\title{
Preliminary Study of Insertion Device Effect on Dynamic Aperture using RACETRACK
}

\author{
Yong-chul Chae* and E. A. Crosbie \\ APS, Argonne National Laboratory
}

May 8, 1992

\begin{abstract}
We studied the effects of an insertion device (ID) on the dynamic aperture using the new version of RACETRACK. We found that the nonlinear effect of the ID is the dominant effect on the dynamic aperture reduction compared to the other multipole errors which exist in the otherwise ideal lattice. The previous study of dynamic aperture was based on the assumption that the effect of the fast oscillating terms in L. Smith's Hamiltonian is small, and hence can be neglected in the simulation. The remarkable agreement between the previous study and the current results using RACETRACK, including all effects of the fast oscillating terms, justfied those assumptions at least for the APS ring.
\end{abstract}

\section{DISCLAIMER}

This report was prepared as an account of work sponsored by an agency of the United States Government. Neither the United States Government nor any agency thereof, nor any of their employees, makes any warranty, express or implied, or assumes any legal liability or responsibility for the accuracy, completeness, or usefulness of any information, apparatus, product, or process disclosed, or represents that its use would not infringe privately owned rights. Reference herein to any specific commercial product, process, or service by trade name, trademark, manufacturer, or otherwise does not necessarily constitute or imply its endorsement, recommendation, or favoring by the United States Government or any agency thereof. The views and opinions of authors expressed herein do not necessarily state or reflect those of the United States Government or any agency thereof.

"On leave from Physics Department, University of Houston. 


\section{DISCLAIMER}

Portions of this document may be illegible in electronic image products. Images are produced from the best available original document. 


\section{Introduction}

This report describes a study of the effect of insertion devices on reduction of dynamic aperture. The field components for a planar insertion device (ID) suggested by K. Halbach [1] are given by:

$$
\begin{aligned}
& B_{y}=B_{o} \cosh k_{x} x \cosh k_{y} y \cos k z \\
& B_{x}=\frac{k_{x}}{k_{y}} B_{o} \sinh k_{x} x \sinh k_{y} y \operatorname{cosk} z \\
& B_{z}=-\frac{k}{k_{y}} B_{o} \cosh k_{x} x \sinh k_{y} y \operatorname{sink} z
\end{aligned}
$$

where

$$
k_{x}^{2}+k_{y}^{2}=k^{2}=\left(\frac{2 \pi}{\lambda}\right)^{2}
$$

and $\lambda$ is the period length.

The Hamiltonian with respect to the reference orbit suggested by L. Smith [2] is given by:

$$
\begin{aligned}
H= & \frac{1}{2}\left(p_{x}^{2}+p_{y}^{2}\right)+\frac{1}{4 k^{2} \rho^{2}}\left[\cosh ^{2} k_{x} x \cosh ^{2} k_{y} y+\frac{k_{x}^{2}}{k_{y}^{2}} \sinh ^{2} k_{x} x \sinh ^{2} k_{y} y\right] \\
& -\frac{\operatorname{sinks}}{k \rho}\left[p_{x}\left(\cosh k_{x} x \cosh k_{y} y-1\right)-\frac{k_{x}}{k_{y}} p_{y} \sinh k_{x} \sinh k_{y} y\right] .
\end{aligned}
$$

Expanding to fourth order in $x$ and $y$,

$$
\begin{aligned}
H= & \frac{1}{2}\left(p_{x}^{2}+p_{y}^{2}\right)+\frac{1}{4 k^{2} \rho^{2}}\left(k_{x}^{2} x^{2}+k_{y}^{2} y^{2}\right)+\frac{1}{12 k^{2} \rho^{2}}\left[k_{x}^{4} x^{4}+k_{y}^{4} y^{4}+3 k_{x}^{2} k^{2} x^{2} y^{2}\right] \\
& -\frac{s i n k s}{2 k \rho}\left[p_{x}\left(k_{x}^{2} x^{2}+k_{y}^{2} y^{2}\right)-2 k_{x}^{2} p_{y} x y\right] .
\end{aligned}
$$

For $k_{x}=0$, i.e. an ID with infinite width in the horizontal direction, this Hamiltonian has a constant linear focusing term and a octupole like term in the vertical direction, if we neglect the fast oscillating (compared to betatron oscillation) term contained in $\sin (k s)$. Neglecting the $\sin (k s)$ term, we may treat the ID as a long quadrupole with a lumped nonlinear element at the center. This approximation makes a sense if the tune shift from the fast oscillating term is small, which is true for the APS lattice because the variation of beta function is small in the ID section. The previous tracking study based on this approach was done by Ed Crosbie using his own PACMAN code [3]. He showed that the dynamic aperture was larger than the physical aperture at the ID section.

As a continuation of Crosbie's work to include the effect of the fast oscillating term, we used the new version of RACETRACK [4] for the dynamic aperture study. 
RACETRACK provides two methods to integrate the equation of motion through ID fields, both of which satisfy the symplectic condition. One method uses the canonical integration scheme originally developed by.R. Ruth (called the Ruth scheme [5] in this report) and the other uses the one developed at BESSY (the BESSY scheme [6]).

\section{Comparison of Ruth and BESSY Schemes}

Although the two integration methods (Ruth and BESSY schemes) adopted in RACETRACK $[7,8]$ are very different, from the user's point of view the difference between the two is that in the Ruth scheme the integrating step size along the beam direction, $d z$, should always be less than one period length, $\lambda$, of the insertion device, whereas in the BESSY scheme $d z$ could be an integral multiple of $\lambda$. In both schemes we should use a small enough step size to achieve sufficient accuracy. Since the integration through the ID is time consuming, if the two methods yield the same accuracy, we will clearly prefer the faster one.

A convergence test for the two methods was performed. For this test, a single Type-A undulator was installed in the APS ring. The parameters for a Type-A undulator are: period length $=3.3 \mathrm{~cm}$, number of periods $=70$ and peak field $=$ 0.85 Tesla. With the initial conditions of $x=2 \mathrm{~cm}$ and $y=1 \mathrm{~cm}$ at the injection point, we tracked a particle 200 turns and the final horizontal positions were compared for different step sizes. The results from the two methods are shown in Fig. 1. Both methods showed convergence as the number of steps per period were increased, to 60 for the Ruth scheme and to 2 for the BESSY scheme. A CPU time comparison showed that the BESSY scheme was faster than the Ruth scheme by a factor of five. Hence we used the BESSY scheme for our dynamic aperture study.

\section{Effects of ID on Dynamic Aperture}

For $k_{x}=0$, the Hamiltonian in Eq. 3 becomes simplified as:

$$
H=\frac{1}{2}\left(p_{x}^{2}+p_{y}^{2}\right)+\frac{1}{2}\left(\frac{1}{2 \rho^{2}}-\frac{p_{x} k}{\rho} \operatorname{sinks}\right) y^{2}+\frac{k^{2}}{12 \rho^{2}} y^{4}+\frac{k^{4}}{90 \rho^{2}} y^{6}+H . O .
$$

The constant linear vertical focusing strength in this Hamiltonian, for a Type-A undulator, is:

$$
\frac{L_{I D}}{2 \rho^{2}}=0.0015\left(m^{-1}\right)<<K_{Q}=0.511\left(m^{-1}\right),
$$


where $K_{Q}$ is the typical quad strength in the APS ring. Hence the linear effect of an ID considered here will be very small. This is verified in Fig. 2 which shows that dynamic aperture reduction is mainly due to the nonlinear effect of an $\mathrm{ID}$. We investigated the effect of the fast oscillating linear term. We noted that, for $k_{x}=0$, the horizontal canonical momentum, $p_{x}$, was constant, hence the linear equation of motion became the Mathieu equation, and we found that additional tune shift was negligible within the wide range of $p_{x}$.

Since the lattice elements in the ring have error fields due to the imperfect construction, the effects of the multipole errors in the lattice were investigated. For this purpose, the random normal sextupole and octupole field errors based on the APS magnetic field tolerance budget were included in the dipole and quadrupole magnets. The rms values of these multipole errors are $b_{2 D}=5 \times 10^{-5}, b_{3 D}=1 \times 10^{-5}$, $b_{2 Q}=1 \times 10^{-4}$ and $b_{3 Q}=5 \times 10^{-5}$ where $b_{n D}$ means the normal $2(n+1)$-pole field coefficient in the dipole magnets [9]. Although these error fields reduce the dynamic aperture of an ideal lattice without an ID, we expected that, because ID effects on dynamic aperture reduction are so dominant, the inclusion of the multipole errors in the ordinary lattice would not cause much additional change to a dynamic aperture already reduced by the presence of the ID. This is verified in Fig. 3. For this reason, all the results presented in this report were obtained with the ideal lattice.

We also investigated the effects of an ID with nonzero $k_{x}$. For $k_{x}=0$, dynamic aperture reduction is mainly in the vertical direction as expected, but we might expect an additional reduction in horizontal axis for nonzero $k_{x}$. This is shown in Fig. 4. Note that $k_{x}=10$ and $20 \mathrm{~m}^{-1}$ corresponds to $k_{x} / k_{y}=0.0526$ and 0.106 , respectively, for a Type-A undulator with $\lambda=3.3 \mathrm{~cm}$. Although $k_{x}=0$ is a good approximation for a realistic planar undulator, Fig. 4 shows that values of $k_{x} / k_{y}$ as large as $10 \%$ do not effect the dynamic aperture.

The results for multiple IDs are shown in Fig. 5. As expected, there is futher reduction in the dynamic aperture when the number of IDs around the ring is increased, however, it does not collapse to smaller than the physical vacuum chamber aperture.

\section{Comparison of the Results from PACMAN and RACETRACK}

During this study, we compared PACMAN and RACETRACK for the different types of ID effects and found remarkably good agreement between the two. We present here a few results from the two programs side by side. Figure 6 shows the very good agreement between the two programs for dynamic aperture results with a single Type-A undulator in the APS ring. Because PACMAN can approximate an ID as 
an elongated quadrupole with the arbitrary nonlinear multipoles at the center, we compared the total nonlinear effects of the ID with the effects of an octupole-like term only, shown in Fig. 6-a. It is clear that the octupole term alone cannot represent well the total effect of the ID.

In order to understand the effects of each multipole contained in the expanded Hamiltonian in Eq. (4), we studied the amplitude-dependent tune shift from the ID. For this purpose, we turned off all the ordinary nonlinear elements in the ring (the sextupoles in the APS ring) and recorded the vertical tunes for the different vertical amplitudes. Since horizontal amplitudes are fixed as zero, the horizontal tune is the same as the linear tune of the ring (this is not exactly true because the fast oscillating term in the Hamiltonian couples the horizontal momentum with the vertical coodinates, but the resulting horizontal tune changes are negligible within the three significant figures). The result from RACETRACK is shown in Fig. 7-a. Letting $\Delta \nu_{y}=a J_{y}+b J_{y}^{2}$, we compared the coefficients of $a$ and $b$ obtained by the analytic estimation and the curve fit of the numerical results below as,

$$
\begin{aligned}
& \Delta \nu_{y}=+0.443 J_{y}+534.56 J_{y}^{2} \text { (Analytic) } \\
& \Delta \nu_{y}=-0.750 J_{y}+518.20 J_{y}^{2} \text { (CurveFit). }
\end{aligned}
$$

Analytic results include the first- and second-order effects of the octupole-like term and the first-order effect of the dudecupole-like term in Eq. (4). Note that the secondorder effect of the octupole on $b$ is as small as -2.44 which is negligible compared to the dudecupole effect. Agreement between the two estimates is very good except that the sign of $a$ from the curve fit is wrong. Because of this quadratic dependence of tune shift on amplitude, and also because within the range of the maximum stable amplitude, $J_{y}=0.0164 \mathrm{~mm}$, the contributions to the tune shift from the octupole and dudecupole are comparable to each other, we may have to include the dudecupole effects in addition to the octupole in order to represent the total nonlinear ID effects well. In Fig. 7-b, we show the amplitude-dependent tunes on the tune diagram. It appears that, with increase in the vertical amplitude, the tune approaches the $\nu_{x}+2 \nu_{y}=n$ resonance line. However, since we do not know the tune at the unstable amplitude, we cannot tell which resonance is driving the particle to be unstable.

In another comparison, we increased the peak field of the Type-A undulator from $0.85 \mathrm{~T}$ to $4.0 \mathrm{~T}$. This particular choice of the peak field was made to simulate the effect of a Type-A undulator on a beam of about $1.5 \mathrm{GeV}$. Again dynamic apertures from the two programs agree very well, as shown in Fig. 8. But the interesting result from PACMAN is that the dynamic aperture reduction from the octupole alone is very close to the whole nonlinear effect. This similarity is also seen in the phase space plot shown in Fig. 9. The three plots in Fig. 9 from PACMAN with the different nonlinear terms are essentially same as the one from RACETRACK.

Finally we show the amplitude-dependent tune for the $4.0 \mathrm{~T}$ case in Fig. 10. This shows that the tune increases more linearly than the $0.85 \mathrm{~T}$ case, which explains why 
the octupole alone represents the total ID effect so well in the dynamic aperture and phase plot. We also show the tune diagram which is similar to Fig. 6-b. Again the tune approaches the $\nu_{x}+2 \nu_{y}=n$ resonance line.

\section{Conclusion and Discussion}

A preliminary study of the effects of insertion devices on the dynamic apereture is presented in this report. We found that for the Type-A undulator considered for the APS project, the nonlinear effect was the dominant cause of dynamic aperture reduction. Also, even with up to $20 \mathrm{IDs}$ in the ring, the maximum stable amplitude of beam oscillation is still greater than the physical vacuum chamber aperture.

We also found that, when we compared the numerical results from RACETRACK with those obtained from Ed Crosbie's PACMAN, agreement between the two was remarkably good. Hence we may conclude that the effects of the fast oscillating term contained in the ID Hamiltonian are small.

\section{Acknowledgements}

We wish to thank Dr. Lee Teng for his numerous suggestions on this problem. Also the discussion with Dr. R. Nagaoka who visited APS from Sincrotrone Trieste was most helpful to us in our understanding of this problem.

\section{References}

1. K. Halbach, "Physical and Optical Properties of Rare Earth Cobalt Magnets," NIM, Vol 187, 1981.

2. L. Smith, "Effects of Wigglers and Undulators on Beam Dynamics," LBL ESG TECH NOTE-24, September, 1986.

3. 7-GeV Advanced Photon Source, Conceptual Design Report, ANL-87-15.

4. F. Iazzourene, et al., "RACETRACK USER'S GUIDE VERSION 4," Sincrotrone Trieste, July, 1991.

5. R. Ruth, "A Canonical Integration Technique," IEEE NS-30, August, 1983.

6. J. Barhdt and G. Wustefeld, "A New Tracking Routine for Particles in Undulator and Wiggler Fields: Part I and II," BESSY TB 158, October, 1990.

7. R. Nagaoka and L. Tosi, "An Improved Scheme for Integrating the Particle Motion through the Insertion Device in RACETRACK," ST/M-90/6, Sincrotrone Trieste, March, 1990. 
8. R. Nagaoka and L. Tosi, "An Extended Scheme of the New Tracking Routine for Nonplanar Insertion Devices," ST/M-91/4, Sincrotrone Trieste, May, 1991.

9. E. Lessner, Private communication, 1992. 


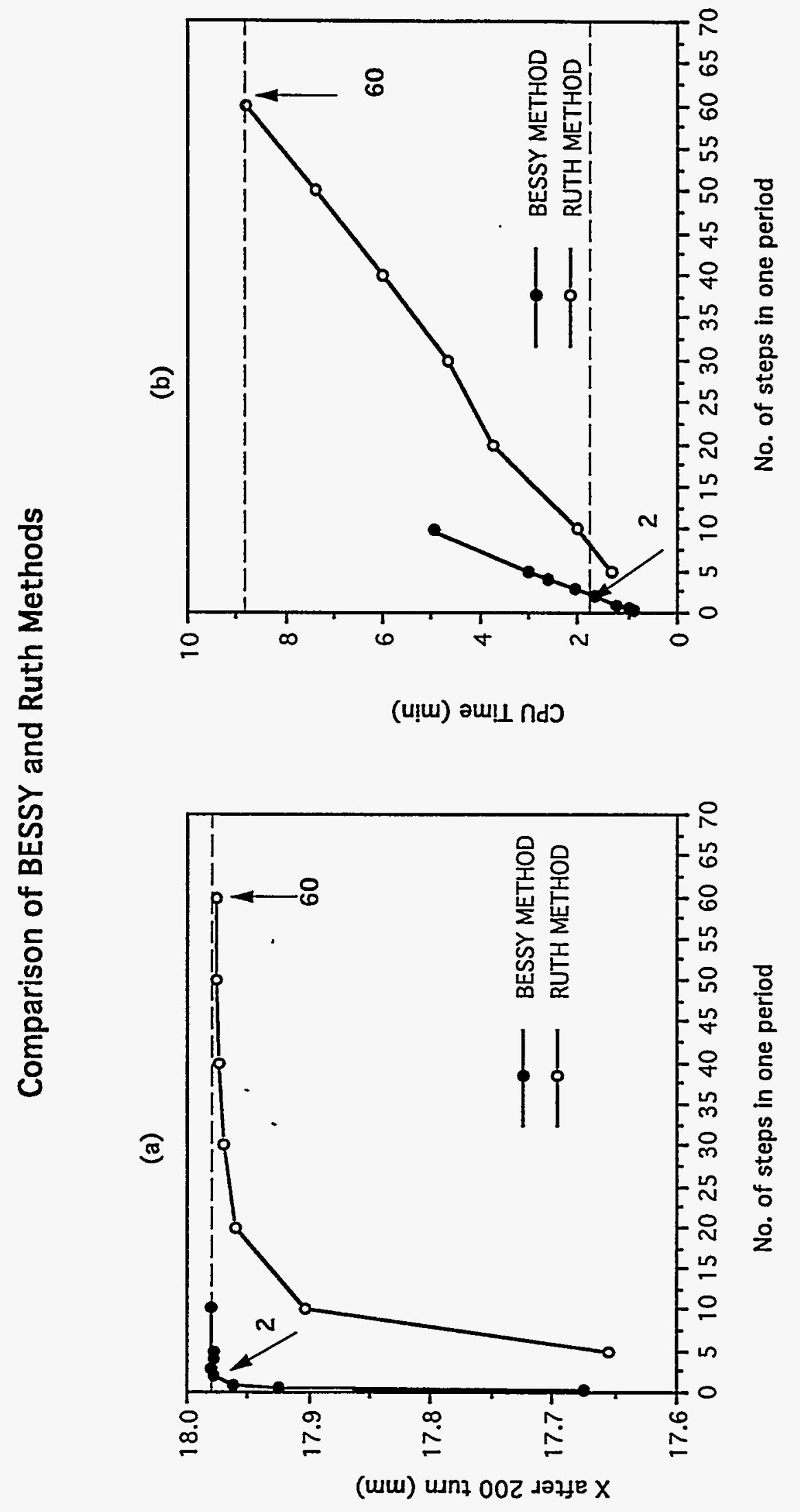

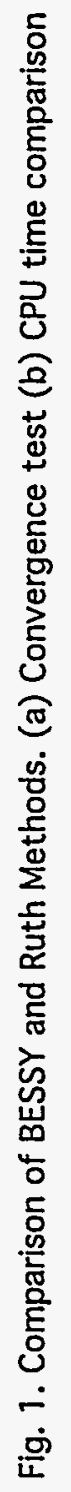




\section{Comparison of Linear and Total Effects of ID on Dynamic Aperture}

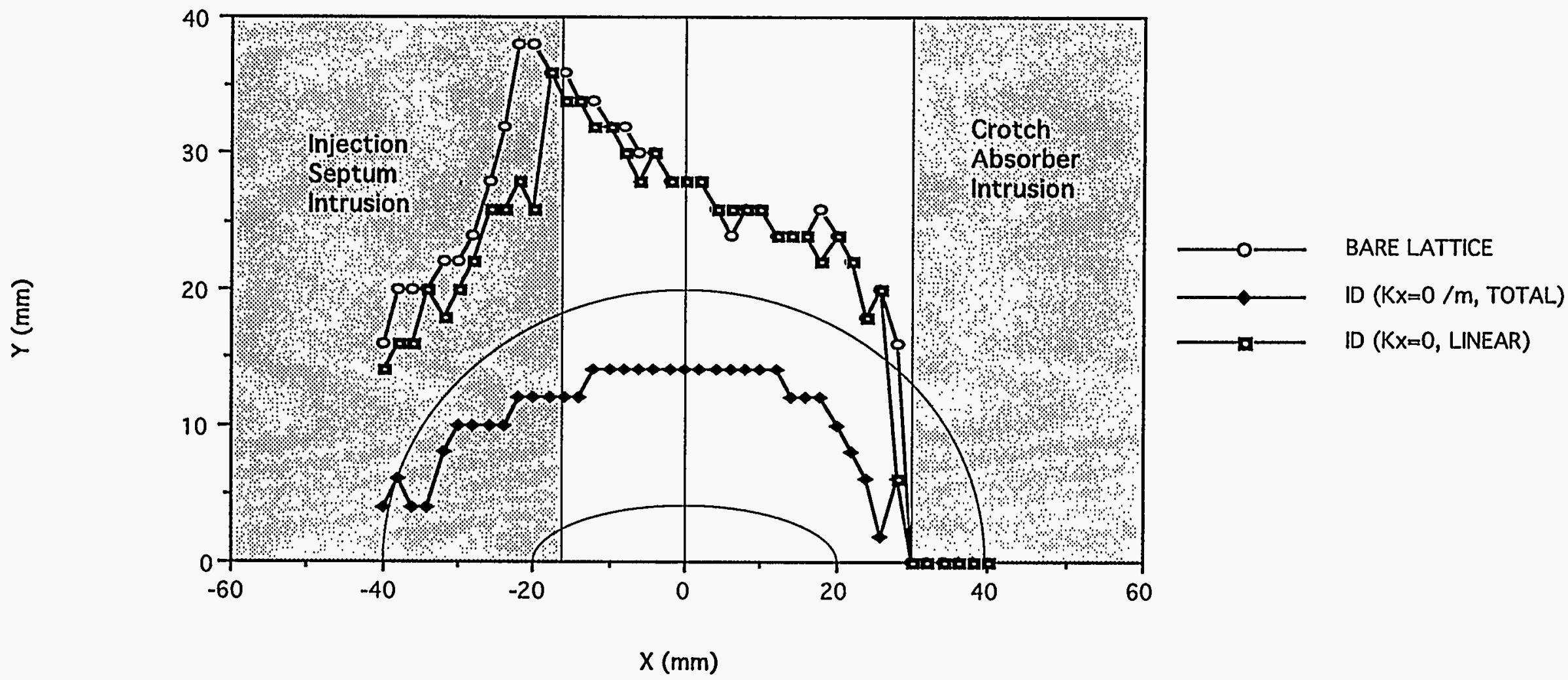

Fig. 2. Dynamic aperture reduction due to a single insertion device (Linear effect v.s. Total effect) Type-A Undulator: Period $=3.3 \mathrm{~cm}$, No. of Periods $=70$, Peak Field $=0.85$ Tesla 
ID Effect on Dynamic Aperture with the Multipole Errors in Lattice

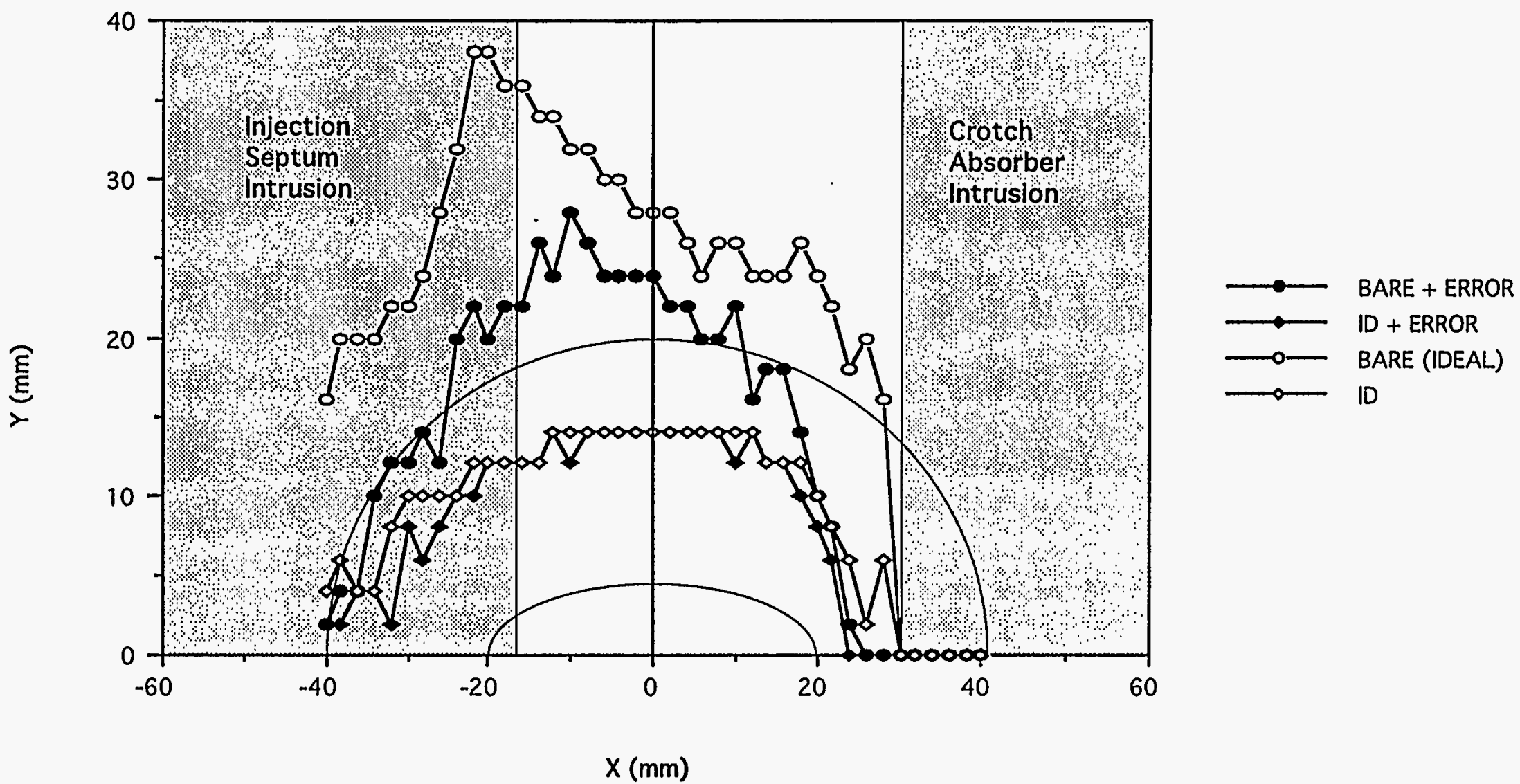

Fig. 3. Dynamic aperture reduction due to a single insertion device with and without the multipole errors in the lattice. Type-A Undulator: Period $=3.3 \mathrm{~cm}$, No. of periods $=70$, Peak field $=0.85$ Tesla 


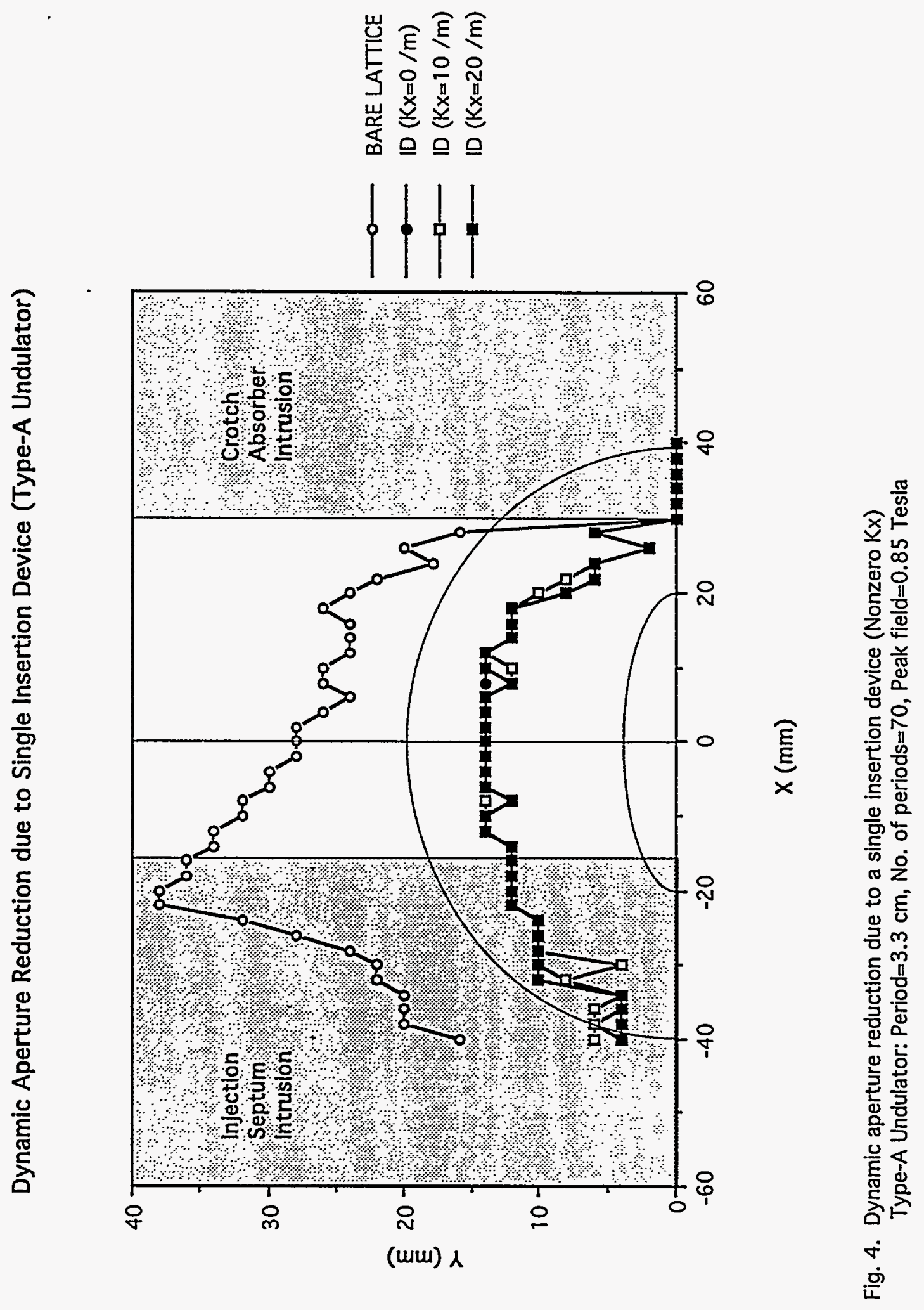


Dynamic Aperture Reduction due to Multiple Insertion Devices (Type-A Undulator)

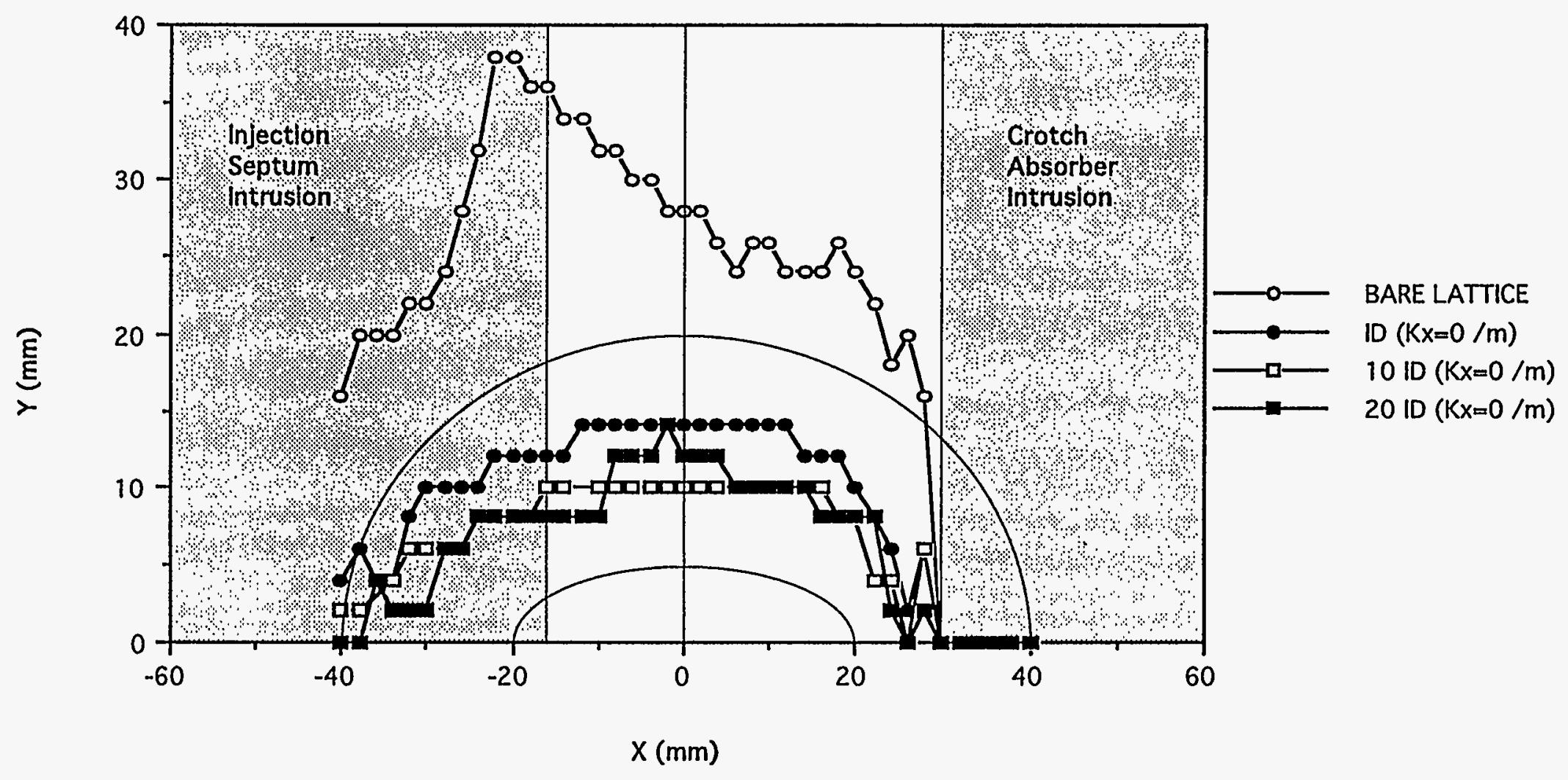

Fig. 5. Dynamic aperture reduction due to multiple insertion device.

Type-A Undulator: Period $=3.3 \mathrm{~cm}$, No. of periods $=70$, Peak field $=0.85$ Tesla 

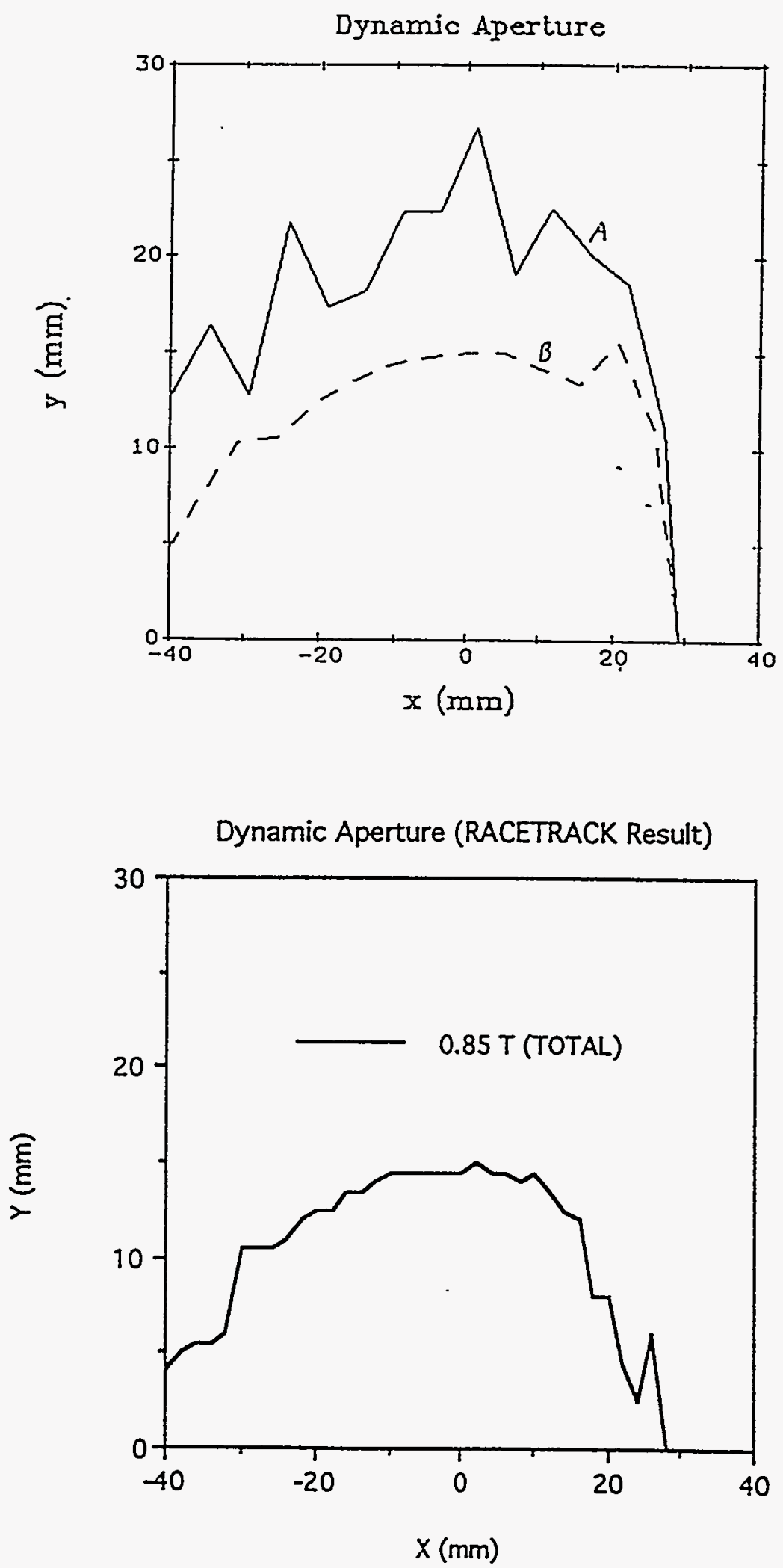

Fig. 6. Dynamic aperture reduction due to $0.85 T$ Undulator TOP: PACMAN (solid line: octupole effect only, dashed line: total effect), BOTTOM: RACETRACK 
(a) Amplitude Dependent Tune Shift

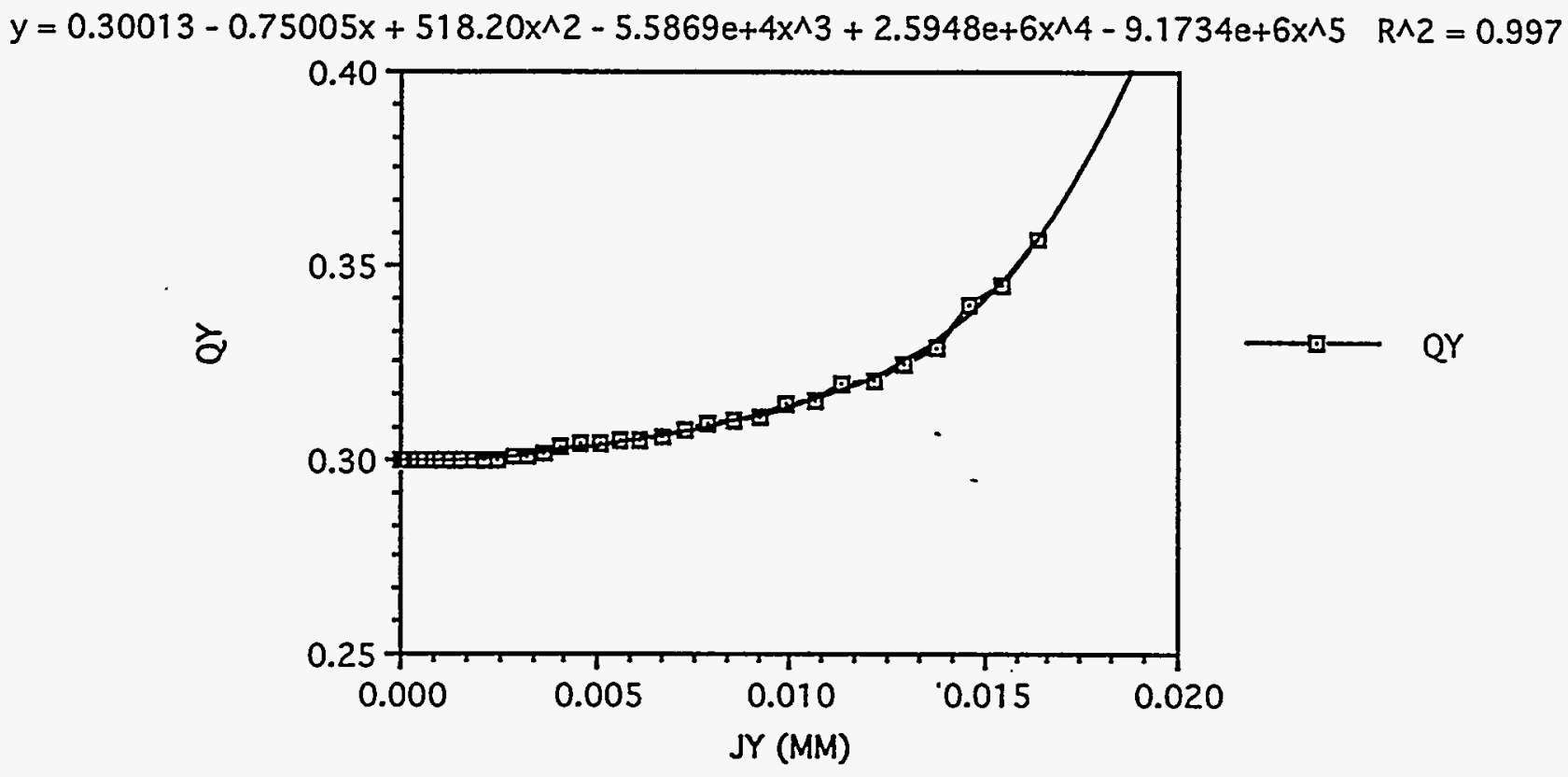

(b) Tune Diagram

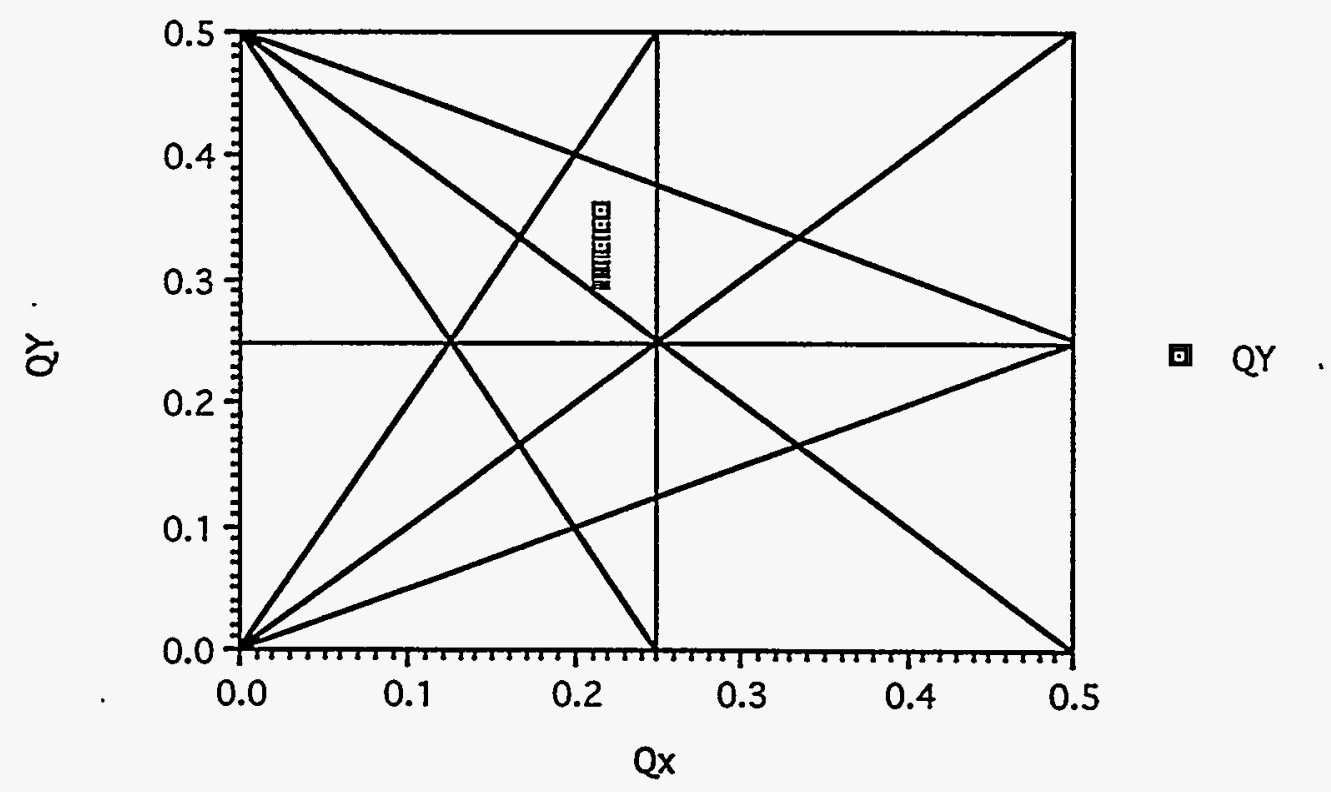

Fig. 7. Amplitude dependent tune shift (0.85 T Undulator) 


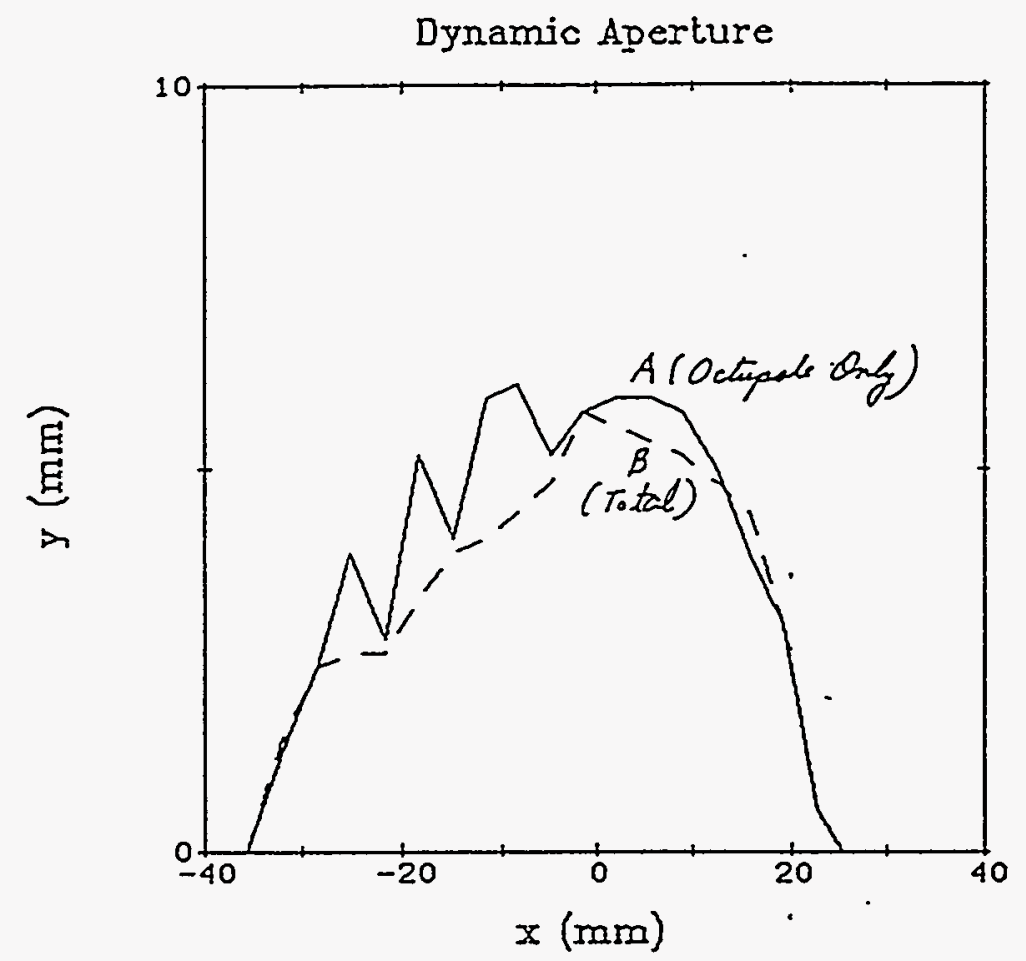

Dynamic Aperture (RACETRACK Result)

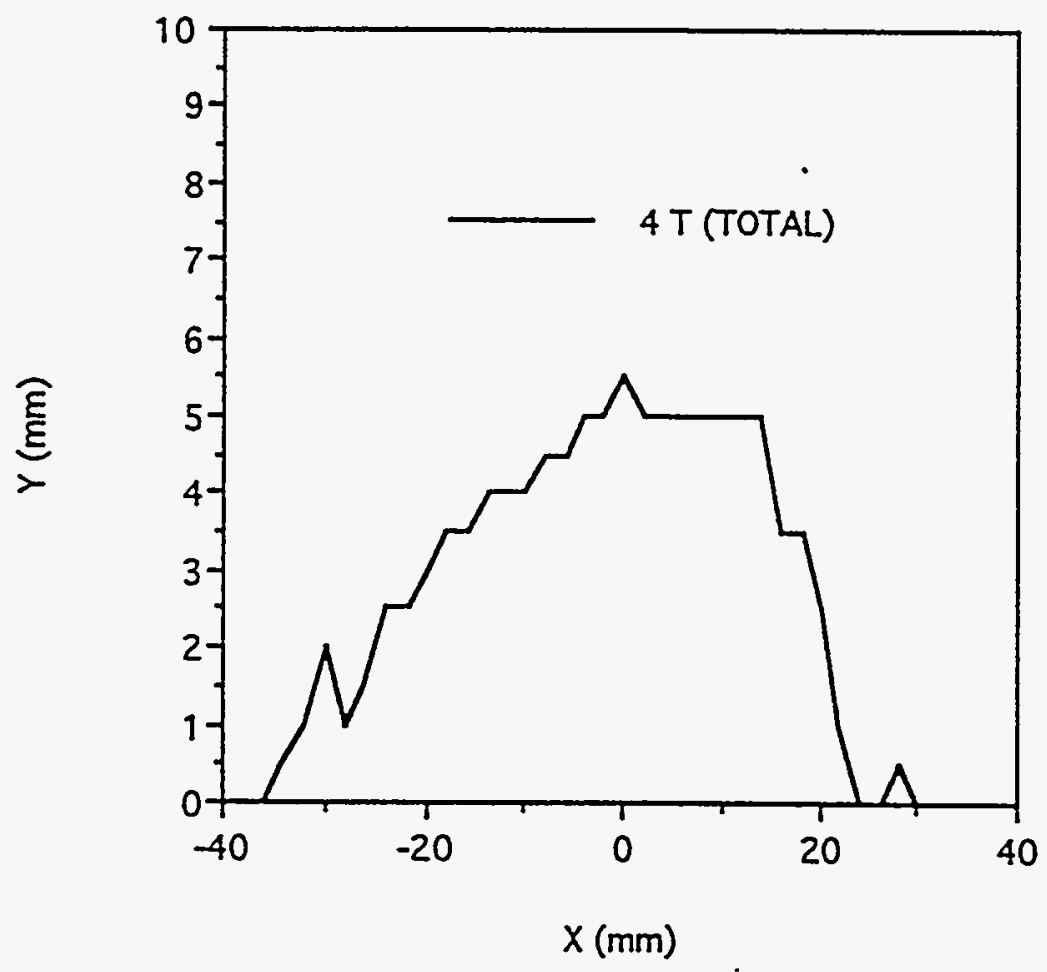

Fig. 8. Dynamic aperture reduction due to $4.0 \mathrm{~T}$ undulator TOP: PACMAN (solid line: octupole effect only, dashed line: total effect), BOTTOM: RACETRACK 


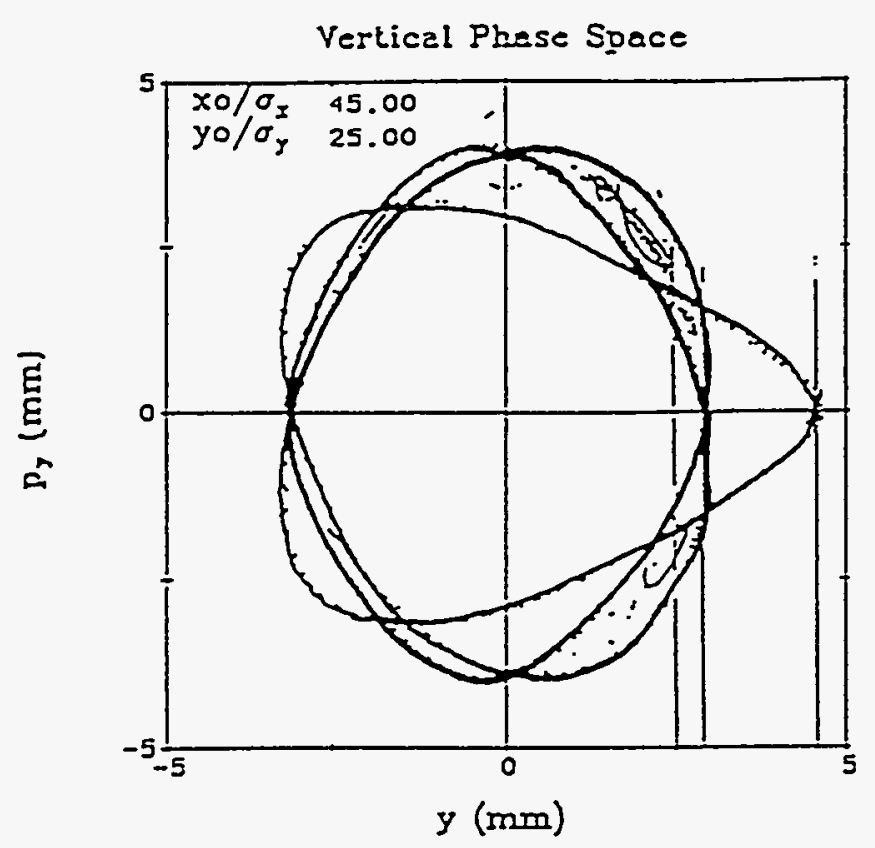

(a)

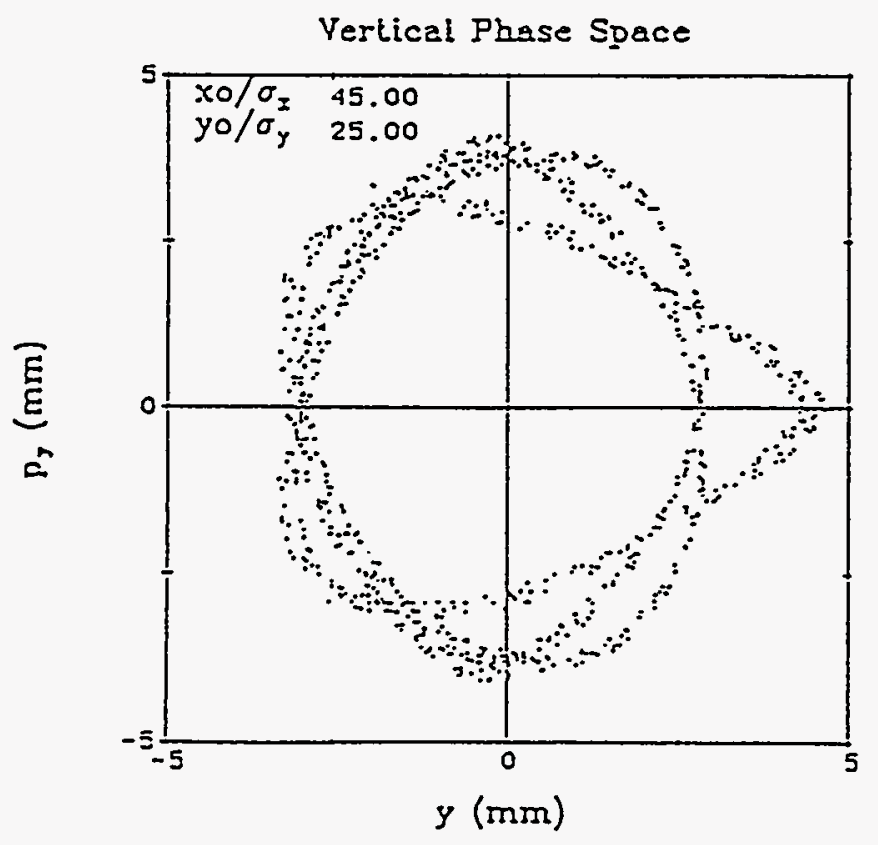

(c)

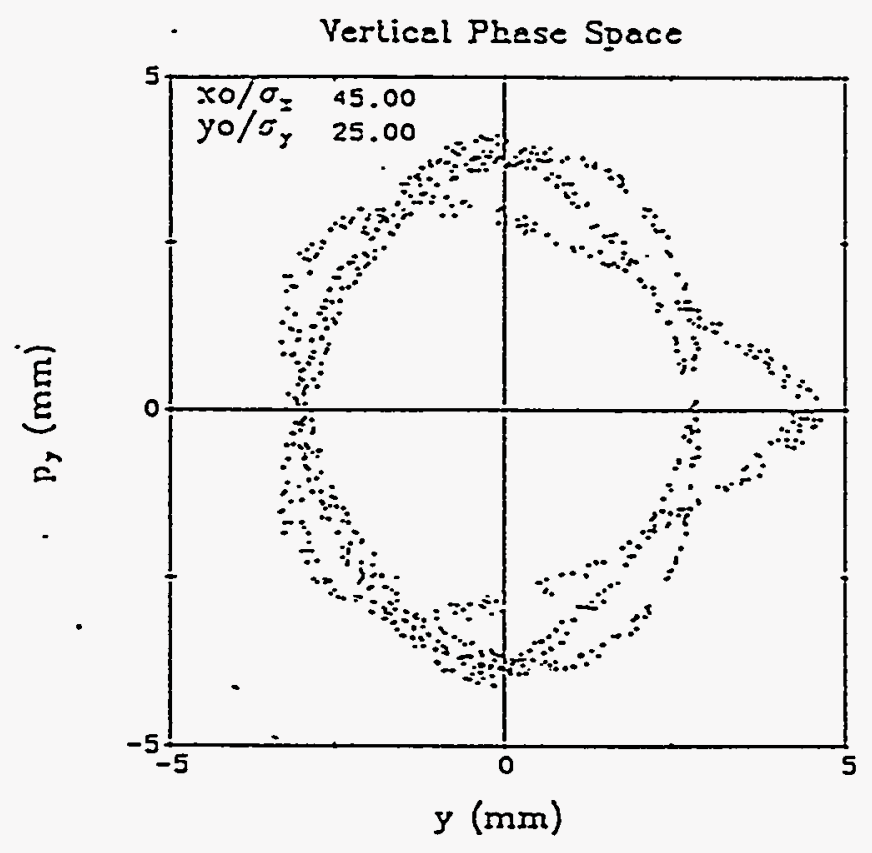

(b)

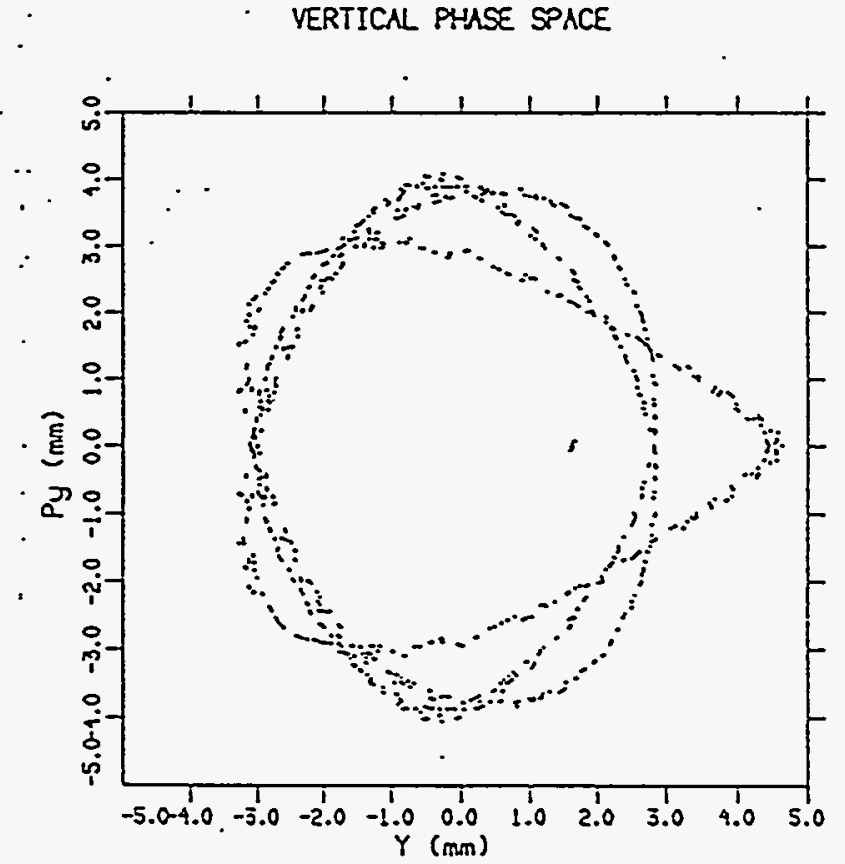

(d)

Fig. 9. Phase space comparison (4 T Undulator)

(a) PACMAN (octupole effect only), (b) PACMAN (octupole plus dudecupole effects only),

(c) PACMAN (total nonlinear effect), (d) RACETRACK 
(a) Amplitude Dependent Tune Shift

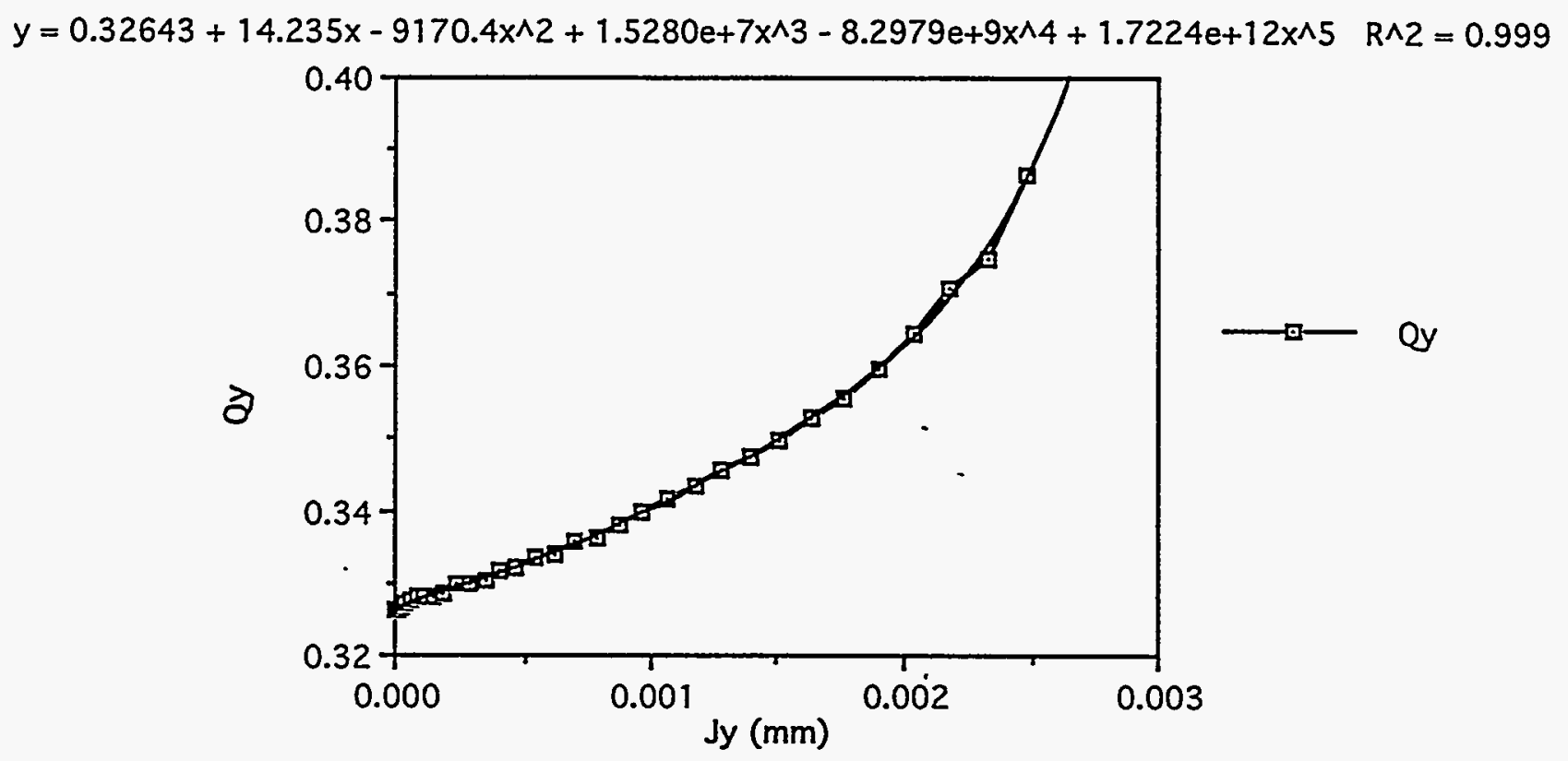

(b) Tune Diagram

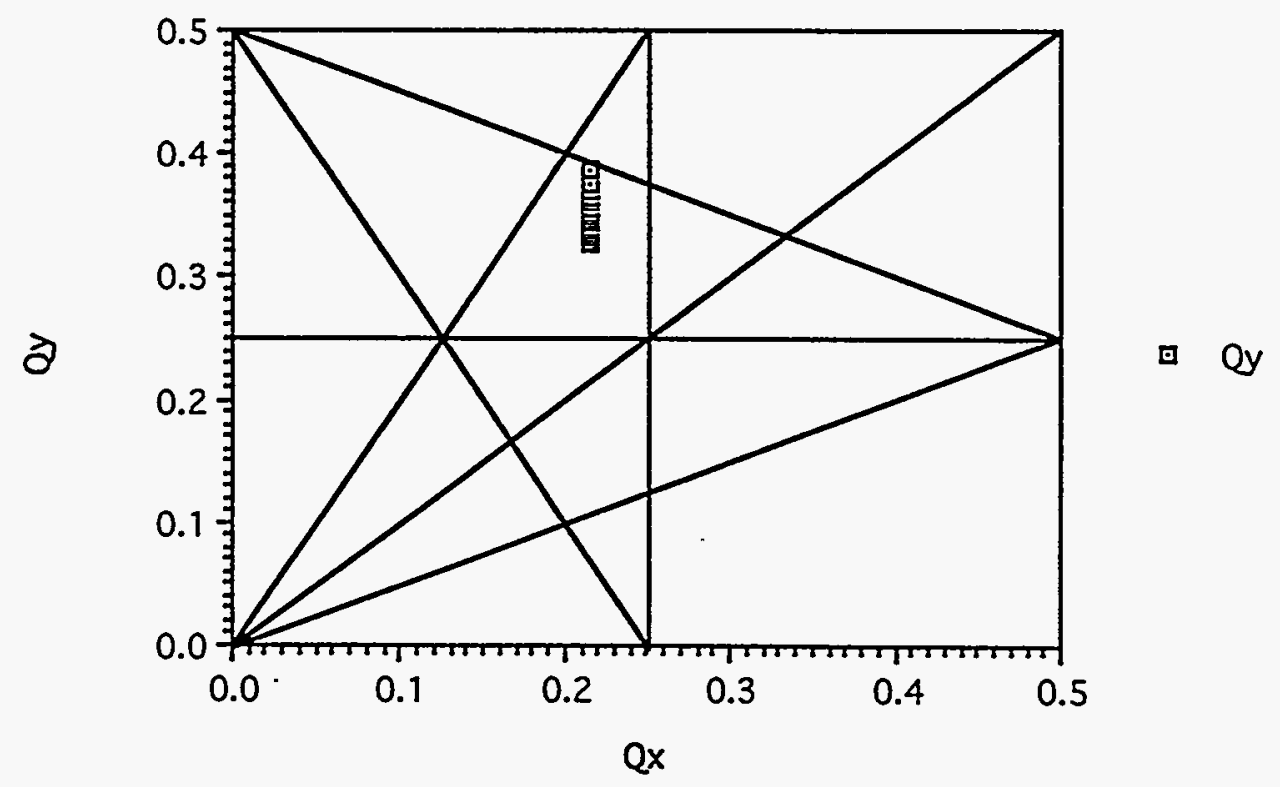

Fig. 10. Amplitude dependent tune shift (4.0 T Undulator) 